wide. They were described by Dr. Seiffert as slender, occasionally compressed, hexagonal pillars with a rhombohedron (Fig. 1). A more detailed description will appear elsewhere. The instability of the crystalline structure is very marked, and the crystals are best kept in the mother liquor in a refrigerator.

The suspension was sent from Berlin to Geneva and kept at $5^{\circ} \mathrm{C}$. Prior to exposure, the specimen was placed in a thin glass tube (Markröhrchen) and this was sealed with wax at both ends. This was necessary because otherwise evaporation takes place during the exposure and the lattice is destroyed. As in the case of the crystalline substances described by Bernal and Wyckoff, water of crystallisation is necessary for the production of an interference pattern by this protein. Exposures were made with copper $K \alpha$-radiation, 15 m.a., $35 \mathrm{kv}$,, at distances up to $77 \mathrm{~mm}$. The temperature was always below $12^{\circ} \mathrm{C}$.; duration of exposure 10-40 hours ; pin-hole collimator $0.7 \mathrm{~mm}$., $80 \mathrm{~mm}$. long. The lines are nevertheless relatively weak.

$\begin{array}{cc}\text { Number } & \text { Spacing (A.) } \\ 1 & 41 \\ 2 & 28 \\ 3 & 25 \\ 4 & 14 \cdot 8 \\ 5 & 11 \cdot 1 \\ 6 & 10 \cdot 1 \\ 7 & \mathbf{4} \cdot 8 \\ 8 & 3 \cdot 4 \\ 9 & \mathbf{2} \cdot 2\end{array}$

$\quad$ Intensity
very strong
medium
weak
weak
medium
medium (diffuse)
medium (diffuse
strong (diffuse
weak (difuse

The spacings and intensities are similar to those obtained by Wyckoff and Corey for oxyhæmoglobin. From the great separation of the planes, it follows that the unit cell is large and accordingly should contain at least one 'chemical' molecule of protein. The molecular weight of Bence-Jones protein as determined by Svedberg is about $\mathbf{3 4 , 0 0 0}$.

A. Magnus-Levy. KuRT H. MEYer.

W. LOTMAR.

Berlin and Geneva.

Feb. 3.

${ }^{1}$ NATURE, 133, $794(1934)$
${ }_{2}$ Science, $81,365(1935)$.

\section{Positive Platinum Sols}

A DETalled investigation on the adsorptive properties and the capacity of platinum electrodes, made by A. Frumkin and A. Slygin ${ }^{1}$, has led to the conclusion that there may exist, besides the negative platinum sol with oxides on the surface of the particles, which has been described by Pennycuick, two further varieties of platinum sols. One of them should be a negative sol, with its surface coated with a film of adsorbed hydrogen, the outer part of the double layer consisting of cations. The other should be a positive sol with a double layer, the outer part of which is formed by anions. According to the results obtained with platinum electrodes, it was expected that positive sols would be obtained by a careful oxidation of negative $\mathrm{H}_{2}$-platinum sols. These positive sols must themselves be transformed by further oxidation into the usual negative sols. These conclusions were announced by A. Frumkin at the discussion on "Colloidal Electrolytes" held by the Faraday Society in September $1934^{2}$; it remained uncertain, however, whether the stability of positive platinum sols, which is determined mainly by an electrical double layer, is such that it would be possible to prepare them.
We had prepared and investigated ${ }^{3}$ negative $\mathrm{H}_{2}$-platinum sols, and we have now succeeded in preparing positive platinum sols by a very slow and careful oxidation of negative $\mathrm{H}_{2}$-platinum sols. The experimental procedure was based on a control of the degree of oxidation of the platinum surface by measurement of the conductance of the sol. On adding a small amount of hydrochloric acid to a $\mathrm{H}_{2}$-platinum sol, there is a rise of conductance of the sol, corresponding to the amount of acid added, as no acid is adsorbed. During the bubbling of oxygen highly diluted with nitrogen through the sol, there is a continuous change of conductance, due to adsorption of acid, followed by its partial desorption when the surface begins to be covered with an oxide film. Each point of the curve corresponds to a definite state of the surface. Stopping the further oxidation at a definite point by saturating the sol with pure nitrogen, and transferring the sol to an apparatus for cataphoretic measurements in an atmosphere of pure nitrogen, we have been able to measure the cataphoretic velocity of sols corresponding to different degrees of oxidation, made all from the same initial batch of $\mathrm{H}_{2}$-platinum sol. The following data are typical of the results obtained :

Cataphoretic mobility Initial $\mathbf{H}_{2}-\mathbf{P t}$-sol During progressive oxidation

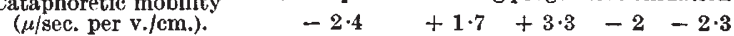

The negative charge of the last portions of sol corresponds to the state of the surface when acid is partly desorbed, which is accounted for by the formation of an oxide film.

The same effects can be obtained with sulphuric acid instead of hydrochloric, but the sol is much more unstable. It is not yet possible to say whether these positive platinum sols can exist for a considerable length of time.

These results show that the particles of a platinum sol behave like small platinum gas electrodes. The existence of positive platinum sols clears up to some extent the contradiction which hitherto existed between data on the sign of the charge of metallic surfaces as deduced from electrokinetic measurements and from electrode potentials.

Nathalie Bach.

N. BALASCHOWA.

Laboratory of Surface Phenomena,

Karpov Chemical Institute,

Moscow.

Feb. 10

1 Sow. Phy8.. 4, 239 (1933) ; C.R. Acad. Sci. U.R.S.S., 2, 176 (1934); Acta Phys. Chim. U.R.S.S., 3, 791 (1935).

Acta Phys. Chim. U.R.S.S., 3, 79 (1935)

\section{The Hydroxyl Bond in $n$-Aliphatic Alcohols}

Robertson ${ }^{1}$, in an interesting note to NATURE, recently pointed out the probable existence of the Bernal-Megaw 'hydroxyl bond' 2 in resorcinol. Further evidence of the existence of such a bond is to be found in a previous paper by E. Ott and the author ${ }^{3}$ on the X-ray study of the $n$-aliphatic alcohols. It was found that in the $\alpha$-modification $\left(\gamma=90^{\circ}\right)$ the distance between hydroxyl groups projected on the $c$-axis is $1.46 \mathrm{~A}$. If a close packing of the hydroxyl heads is assumed, the centres of the $\mathrm{OH}$ groups are then found to be separated by a distance of $3 \cdot 13 \mathrm{~A}$. The value of $a_{0}=4 \cdot 76 \mathrm{~A}$. given by Bernal ${ }^{4}$ is used in this calculation. The distance $3 \cdot 13 \mathrm{~A}$. is less than 